\title{
REFLEXÕES SOBRE O ENSINO DE ENGENHARIA: DESAFIOS NO EXERCÍCIO DA DOCÊNCIA
}

\author{
REFLECTIONS ON ENGINEERING TEACHING: CHALLENGES IN TEACHING
}

Gustavo Yoshio Matsubara ${ }^{1}$, Tayza Cristina Nogueira Rossini ${ }^{2}$

DOI: 10.37702/REE2236-0158.v39p412-419.2020

\begin{abstract}
RESUMO
O avanço tecnológico acontece de maneira cada vez mais acelerado e, com isso, os novos profissionais precisam se adaptar continuamente. $\mathrm{Na}$ área de ensino, para que o sistema de educação tenha capacidade de mudança é necessário levantar reflexões sobre como é estruturado o sistema de ensino tradicional da engenharia brasileira. Além disso, é importante que os docentes estejam atentos às metodologias alternativas de ensino existentes para que sejam utilizadas como ferramentas para o melhor exercício da docência. No presente artigo, serão apresentadas algumas reflexões com relação à situação da profissão docente, dificuldades enfrentadas, além de mencionar estudos de casos de metodologias alternativas presentes na bibliografia aplicadas em cursos de Engenharia.
\end{abstract}

Palavras-chave: Sistema de Ensino de Engenharia; dificuldades na docência; metodologias de ensino alternativas.

\begin{abstract}
The technological advancement requires new professionals and they need to adapt continuously. In order to adequate to these new changes, it is necessary to raise reflections on the roots of the Brazilian teaching practices in engineering. In addition, it is important that professors know the alternative teaching methodologies used as tools for the best teaching practices. In this article, there are some reflections regarding the situation of the teaching profession and some studies of alternative methodologies presented in the bibliography applied in engineering courses.
\end{abstract}

Keywords: Engineering education; teaching difficulties; alternative teaching methodologies.

\section{INTRODUÇÃO}

A reflexão sobre o Ensino em Engenharia deve ser levantada e atualizada constantemente para adaptar os novos profissionais aos desafios que surgem a todo momento no mercado. $\mathrm{O}$ ensino tradicional é baseado em aulas puramente expositivas, por isso, essa metodologia deve ser repensada para que o aluno não tome uma postura tão passiva com relação às informações a serem absorvidas.

As disciplinas presentes nas grades curriculares dos cursos de Engenharia visam a desenvolver capacidades técnicas, interpessoais e de gestão. Entretanto, os métodos de ensino puramente expositivos dificultam o

\footnotetext{
${ }^{1}$ Doutorando em Estruturas e Materiais aplicados à Engenharia Civil - COPPE/UFRJ, gustavoyoshio@coc.ufrj.br.

${ }^{2}$ Doutoranda e Mestre na linha de pesquisa Literatura e Construção de Identidades pela Universidade Estadual de Maringá (UEM). Professora da Graduação (Presencial) e orientadora de trabalhos de conclusão de curso (TCC) de Pós-graduação do Centro Universitário de Maringá (UniCesumar) e professora do curso de Letras da Universidade Estadual de Maringá (UEM). É avaliadora do BASIS (Instituto Nacional de Estudos e Pesquisas Anísio Teixeira - INEP), tayza.rossini@unicesumar.edu.br.
} 
desenvolvimento de tais habilidades. Para isso, rever as metodologias de ensino se torna cada vez mais necessário para que se consiga tentar estimular todas essas características na sala de aula.

De forma a iniciar uma reflexão sobre a necessidade da mudança do sistema de ensino tradicional, o presente artigo aborda como é estruturado o sistema de ensino tradicional, fatores relacionados ao desempenho acadêmico dos estudantes, as dificuldades enfrentadas pelos docentes, as demandas dos novos profissionais e, por fim, apresenta alguns estudos de pesquisadores que verificaram a eficácia de métodos alternativos no ensino de engenharia.

Enfatiza-se que há a necessidade do incentivo de mais pesquisadores na área de engenharia para que seja possível uma maior eficiência do ensino e, consequentemente, que se obtenha a formação dos docentes com maior qualidade.

\section{DISCUSSÕES SOBRE O ENSINO TRADICIONAL DE ENGENHARIA}

Masetto (2012) recorda que em 1970, para se tornar professor, era necessário apenas o bacharelado para o exercício competente da profissão. Esse cenário sofreu alterações a partir de 1980, exigindo-se adicionalmente um curso de especialização. A transformação das obrigatoriedades para a carreira docente se mostrou de maneira contínua, sendo exigida nos dias atuais a formação de mestrado e doutorado. Esses requisitos estão embasados na crença de que se a pessoa tem o conhecimento, automaticamente ela saberá ensinar.

A porta de entrada para a carreira universitária está em grande parte pautada em atividades relacionadas à pesquisa e em uma parcela menor relacionada à docência. Bazzo (2008) destaca, ainda, que a pesquisa possui uma relação mais abstrata e racional, enquanto o ensino apresenta adicionalmente 0 envolvimento humano. $\mathrm{O}$ equilíbrio entre essas características no exercício da docência pode impactar de forma positiva a experiência acadêmica dos alunos.
De forma complementar, pesquisas realizadas por Furtado (2013) constatam que a maioria dos docentes analisados na área de engenharia não possuíam formação específica para o ensino durante o curso de mestrado, sendo que a maioria obteve sua formação de professor no dia a dia da sala de aula. Os docentes que apresentavam alguma especialização na área de educação tiveram essa iniciativa por conta própria.

A especialização por parte dos docentes na área de educação instrui estes sobre as melhores técnicas e alternativas existentes para proporcionar aos alunos um aprendizado mais sólido. Essas técnicas e metodologias, quando aplicadas da maneira correta, refletem em alunos mais motivados e com maior iniciativa no seu próprio aprendizado.

Apesar dos pontos positivos relacionados às novas metodologias e técnicas de ensino, se essas metodologias não forem aplicadas da maneira correta, resultados adversos podem ser obtidos. Seno e Belhot (2009) enfatizam que a não compatibilidade com o estilo de aprendizagem do aluno pode ocasionar uma desmotivação nestes, acarretando resultados academicamente negativos e, consequentemente, frustação até mesmo por parte dos professores.

De forma a se obter uma melhor eficiência no processo de ensino e aprendizagem, os docentes precisam ser verdadeiros estrategistas, sendo necessário estudar, selecionar, organizar e propor melhores ferramentas para que os estudantes se apropriem dos conhecimentos (FURTADO, 2013).

\section{Fatores relacionados ao desempenho dos estudantes}

Monteiro et al. (2009) analisam que características comuns de estudantes que apresentam bom rendimento acadêmico estão relacionadas em grande parte à disciplina e à motivação. Essas características são influenciadas pelas capacidades cognitivas, não-cognitivas (socioemocionais) e estado de saúde mental dos alunos.

As capacidades cognitivas estão em grande parte baseadas na bagagem acadêmica do 
aluno. Essas capacidades estão relacionadas com a habilidade de interpretação, memorização, reconhecimento de padrões, conceituação e racionalização.

Em contrapartida, as capacidades cognitivas (socioemocionais) não necessitam necessariamente de uma bagagem acadêmica para serem desenvolvidas. As capacidades socioemocionais possuem grande ligação com características de persistência, autodisciplina, curiosidade e resiliência (FRAGA, 2015).

$\mathrm{O}$ ensino tradicional é baseado, na sua maior parte, no desenvolvimento das capacidades cognitivas, sendo pouco exploradas as capacidades não cognitivas. Apesar disso, as capacidades não cognitivas desempenham papel importante para o sucesso acadêmico.

Para Achkar et al. (2019), a consequência da não obtenção do sucesso acadêmico pode ter reflexos na vida pessoal e profissional dos alunos, podendo ocorrer o desenvolvimento de sintomas de ansiedade e depressão. O resultado é um aumento do sofrimento para continuação do percurso acadêmico, acarretando uma piora cada vez mais acentuada da situação acadêmica, profissional e pessoal do discente.

Bezerra (2018) analisou a saúde mental dos alunos de 11 cursos de Engenharia do Centro de Tecnologia da Universidade Federal do Ceará (CT-UFC) e foi verificado que $41,9 \%$ deles apresentavam sintomas depressivos, sendo que $6 \%$ apresentavam sintomas graves. Esses resultados apontam e alertam sobre a importância de uma maior atenção no acompanhamento psicológico dos acadêmicos.

\section{Dificuldades docentes no ensino de Engenharia}

Apesar da atribuição de uma parcela da responsabilidade pelo desempenho dos alunos aos docentes, alguns outros fatores devem ser analisados. Como já discutido, os docentes em muitos casos são avaliados com critérios relacionadas à produção acadêmica, sendo muitas vezes a parte pedagógica negligenciada (SEVERINO, 2013). Por isso, muitos docentes priorizam a pesquisa ao invés da docência, devido ao maior reconhecimento profissional oferecido pela área de pesquisa.
A criação de políticas de incentivo à melhoria do desempenho da função docente é essencial para $\mathrm{o}$ processo de ensino $\mathrm{e}$ aprendizagem. Estudos realizados por Achkar et al. (2019) mostram que alunos que tiveram contato com professores com exaustão profissional ou com baixa realização profissional tendem a apresentar resultados acadêmicos ruins.

Além disso, a falta de incentivo e conscientização dos docentes na área de engenharia para o desenvolvimento de atividades relacionadas ao aprofundamento do conhecimento em ensino se torna um dos fatores primordiais para a disseminação do método tradicional e, como já discutido, pode ocasionar resultados pouco satisfatórios no aprendizado dos alunos, além de gerar um descontentamento com a carreira escolhida por parte de alunos e professores.

Adicionalmente a essas adversidades, temse a deficiência de grande parte dos estudantes na área de matemática, oriunda do Ensino Fundamental e Médio. Segundo dados divulgados em 2017 pelo Sistema de Avaliação da Educação Básica (SAEB), apenas 4,52\% dos estudantes analisados do Ensino Médio apresentaram níveis adequados em Matemática. Essa deficiência tem reflexo no desempenho dos alunos do Ensino Superior nas áreas de Exatas, como constatado por Passos et al. (2007), Vidal e Cunha (2019) e Saccaro, França e Jacinto (2019).

Almeida e Godoy (2016) reforçam que as maiores causas de evasão nos cursos de Engenharia estão relacionadas às deficiências na formação da educação básica dos estudantes, às metodologias inadequadas didáticopedagógicas adotadas por parte dos professores e ao acúmulo de reprovações sucessivas durante o percurso acadêmico. Saccaro, França e Jacinto (2019) complementam que, entre 2009 e 2014, o INEP divulgou que os cursos de Exatas, como Matemática, Engenharia Metalúrgica e Mecânica, apresentaram evasão próximas de $90 \%$ e $60 \%$, respectivamente.

Adicionalmente, Almeida e Godoy (2016) propõem que, para diminuição da taxa de evasão, a criação e ampliação de programas de programas de monitoria, tutoria e nivelamento 
para as disciplinas do ciclo básico se tornam boas medidas a serem tomadas.

Os programas de monitorias, tutorias e nivelamentos através de recursos presenciais ou computacionais podem ser ministrados por alunos que apresentam rendimento exemplar. Com isso, auxilia-se os alunos com maiores dificuldades acadêmicas e incentiva-se jovens talentos para a área da docência e pesquisa.

A superação das dificuldades dos alunos nas disciplinas do Ensino Básico (Fundamental e Médio) e do ciclo básico das engenharias é essencial para o docente desempenhar uma melhor dinâmica das aulas. A falta de dinâmica durante a abordagem dos conteúdos acarreta uma diminuição na qualidade das aulas devido à dificuldade do cumprimento da ementa proposta das disciplinas.

O reflexo da diminuição da qualidade das aulas é sentido no decorrer do curso, visto que grande parte das disciplinas ministradas têm forte ligação teórica com as disciplinas ministradas em períodos subsequentes.

Outro fato relevante e que desmotiva grande parte dos alunos é a falta de conexão dos conteúdos abordados com os processos que são utilizados na prática. A dificuldade por parte docente nesse quesito muitas vezes pode ser ocasionada pela falta de experiência prática destes. A ocorrência desse fenômeno pode ser embasada na pouca valorização que o meio prático atribui aos mestres e doutores nos seus processos de recrutamento (SILVA FILHO, 2012 apud MOLISANI, 2017). Além disso, grande parte dos alunos de mestrado e doutorado trabalham em regime de dedicação exclusiva, impossibilitando que estes se insiram no mercado.

Essa falta de valorização da pesquisa acadêmica pelo mercado de trabalho ocasiona uma separação de dois setores que deveriam trabalhar em conjunto para o desenvolvimento do país.

\section{Metodologias Alternativas}

De forma a contribuir com o desenvolvimento e com a readaptação das competências exigidas do profissional de engenharia contemporâneo, vários autores desenvolveram estudos relacionados às metodologias de ensino alternativas frente às aulas puramente expositivas.

Um método conhecido e ainda pouco difundido no Ensino da Engenharia no Brasil é o método PBL (Problem Based Learning). Esse método teve seu início com Barrows e Tamblyn (1976) no curso de Medicina na Universidade de McMaster, localizada no Canadá. O principal diferencial desse método é que o aluno é exposto a um problema proposto e temse o desenvolvimento do conhecimento através de discussões e formulações de hipóteses para tomada de decisões para solução de tal problema.

Apesar do método PBL ter sido inicialmente desenvolvido e voltado para o curso de Medicina, essa metodologia tem sido objeto de estudo de alguns pesquisadores para adaptação na área de Engenharia. Casale, Kuri e Silva (2011) verificaram a eficácia desse método com a aplicação na disciplina de Planejamento e Análise dos Sistemas de Transporte, do curso de Engenharia Civil. Esse método tem grande valor, principalmente em disciplinas que exigem discussões, uma vez que agrega conhecimentos mútuos entre alunos e professores.

Os resultados obtidos por Casale, Kuri e Silva (2011) se basearam na comparação de dois grupos de estudantes. O primeiro grupo exposto ao método de ensino tradicional - e o segundo grupo - exposto ao método de ensino PBL. Os resultados obtidos foram animadores e notou-se um maior comprometimento e responsabilidade por parte dos alunos, que apresentaram um aprendizado de forma mais autônoma. Além disso, os alunos que foram expostos ao método PBL obtiveram uma menor heterogeneidade nas notas e, ainda, apresentaram média superior aos alunos que foram expostos ao método tradicional.

Em contrapartida, Pawson et al. (2006) alertam para alguns cuidados relacionados à preparação dos professores e estudantes, para que ambos estejam alinhados e comprometidos com a mudança de perspectiva do aprendizado proporcionado pelo método, gerando, assim, maior fluidez e eficiência na aquisição do conhecimento. 
Além do método PBL, menciona-se o método de ensino baseado na utilização de laboratórios virtuais. Esses laboratórios possuem uma maior abrangência na acessibilidade dos alunos, podendo ser implementados até mesmo em universidades nas quais não se tem um laboratório adequado devido ao seu alto custo.

A eficácia da utilização dos laboratórios virtuais foi analisada por Guilhermo et al. (2017). Nesse estudo, participaram alunos cursando a disciplina de Mecânica dos Fluidos (Engenharia Civil, Ambiental e de Produção). Com isso, constatou-se o desenvolvimento de competências relacionadas aos conceitos chaves da disciplina.

Ainda, Guilhermo et al. (2017) reportam que a utilização combinada dos laboratórios reais e virtuais gera o melhor desempenho nos testes teóricos, quando este é comparado com o de alunos que utilizaram apenas um dos recursos. Caso a universidade não tenha capacidade de intervir na construção de um laboratório real, devido aos seus altos custos, a utilização de um laboratório virtual supre essa lacuna importante na formação dos alunos.

O desempenho dos alunos com a utilização dos laboratórios virtuais apresentou resultados muito positivos. Os alunos que utilizaram somente o laboratório virtual obtiveram desempenho superior aos alunos que fizeram apenas experiências práticas reais (GUILHERMO et al., 2017).

Outro fato relevante que deve ser utilizado a favor do ensino é o aumento da inclusão digital. Essa inclusão está cada vez mais democrática e o acesso à internet está atingindo cada vez mais pessoas, como reportado por Lavado (2019). Assim sendo, torna-se cada vez mais viável a utilização dessas poderosas ferramentas para melhoria do ensino. Por isso, além dos laboratórios virtuais, o método da aula invertida pode ser potencializado com a utilização dos recursos digitais.

No método da sala de aula invertida, os alunos têm acesso aos conteúdos básicos a serem ministrados pelo professor antes da entrada deste em sala de aula. Esses conteúdos podem ser disponibilizados em forma de videoaulas, apostilas, capítulos de livros, artigos, slides, entre outros. O grande diferencial desse método é que ele é centrado no aluno, sendo o professor apenas um mediador do processo de aprendizagem. Consequentemente, o aluno desenvolve uma maior autonomia na gestão da sua formação.

Pavanelo e Lima (2017) testaram a metodologia da sala de aula invertida na disciplina de Cálculo I nos cursos de Engenharia do ITA. Os autores constataram que os alunos que utilizaram o método obtiveram uma maior média de notas. Entretanto, existe a necessidade de mudanças na postura e na conscientização dos professores e alunos para adequação do método.

Simultaneamente ao desenvolvimento do mundo digital, o mercado de trabalho acompanha essas mudanças que se dão em grande velocidade, para que consiga se manter competitivo. Para tal, poderosas ferramentas são criadas e as universidades devem procurar desenvolver habilidades para adaptar seus alunos a essas constantes mudanças.

Um exemplo de avanço tecnológico que tem se tornado uma tendência no mundo é a utilização do BIM (Building Information Modeling). O BIM é uma tecnologia pela qual se gera, por exemplo, um modelo virtual de uma edificação, de forma precisa e integrada com os vários projetos de uma mesma obra. Esse processo auxilia no suporte necessário para construção, modelagem no ciclo de vida da edificação, tendo impacto na diminuição do custo e no prazo de execução das obras (EASTMAN et al., 2014).

No Brasil, o BIM ainda é muito pouco difundido e poderia ser muito eficaz, por exemplo na fiscalização de obras, gerando uma economia significativa no processo construtivo, como relatado por Miranda e Matos (2015).

Basto e Lordsleem Junior (2016) verificaram a aplicação do ensino do BIM em uma universidade dos EUA. Apesar de se obter uma boa efetividade na abordagem dos conceitos por parte dos alunos, a sua implementação ainda necessita de algumas adaptações e estudos adicionais para ser efetivada no Brasil.

Por fim, através das metodologias de ensino apresentadas até agora, nota-se que a busca contínua por metodologias alternativas de ensino é muito importante para o 
desenvolvimento de capacidades cognitivas e não-cognitivas dos alunos. Contudo, a principal dificuldade provavelmente estará na adaptação dos alunos e docentes na implementação dos novos métodos. Diante disso, é aconselhável que o processo de transição seja feito de forma cautelosa e que passe por uma preparação de conscientização por parte dos alunos e professores.

\section{CONSIDERAÇÕES FINAIS}

O ensino tradicional - baseado em aulas centradas no professor e com aluno apresentando postura passiva com relação aos conteúdos apresentados - está muito enraizado em grande parte das salas de aulas. A necessidade de atualizar, criar e melhorar formas alternativas de ensino se torna cada vez mais evidente. Para isso, é crucial discutir as dificuldades e levantar os fatores responsáveis pela disseminação dessa cultura.

Em grande parte, a disseminação do ensino tradicional está potencializada pelas dificuldades de se desempenhar a carreira docente. Com isso, a dificuldade dos alunos com aprendizado através de métodos pouco efetivos aliada a deficiências do ensino de base (fundamental e médio) tornam esses estudantes possíveis números das estatísticas de evasão dos cursos de graduação.

As possíveis soluções para diminuir as estatísticas de evasão podem ser pautadas na implementação de monitorias e cursos de nivelamento para atender às dificuldades individuais de cada estudante. Adiciona-se, ainda, a necessidade de acompanhamento psicológico dos alunos, com intuito de diminuir e tratar os casos de alunos que apresentam sintomas de ansiedade excessiva e depressão.

Por fim, o presente artigo teve o intuito de levantar algumas reflexões do Ensino da Engenharia no Brasil e, com isso, apresentar algumas alternativas pedagógicas para formar profissionais com maior autonomia e iniciativa para resolver os problemas da sociedade.

Além de tudo, a formação de pesquisadores na área de Educação ainda se mostra discreta em algumas áreas de ensino, como a Engenharia. Por esse motivo, a necessidade de conscientização e incentivo de engenheiros a ingressarem nessa área é fundamental para o desenvolvimento de metodologias cada vez melhores, possibilitando, assim, a formação de profissionais mais preparados para o mercado de trabalho, ensino e pesquisa.

\section{REFERÊNCIAS}

ACHKAR, A. M. N. E. et al. Life Satisfaction and Academic Performance of Elementary School Students. Psico-USF, Bragança Paulista, v. 24, n. 2, p. 323-335, abr./jun. 2019.

ALMEIDA, E.; GODOY, E. V. A evasão nos cursos de engenharia: uma análise a partir do COBENGE. Anais... XLIV Congresso Brasileiro de Educação em Engenharia, Natal, Rio Grande do Norte: COBENGE/UFRN, p. 119, 2016.

BARROWS, H. S.; TAMBLYN, R. An evaluation of problem-based learning in small groups using a simulated patient. Journal of Medical Education, Teerão, v. 51, p. 52-54, jan. 1976.

BASTO, P. E. A.; LORDSLEEM JUNIOR, A. C. Ensino de BIM em curso de graduação em engenharia civil em uma universidade dos EUA: Estudo de caso. Ambiente Construído. Porto Alegre, v. 16, n. 4, p. 45-61, out./dez. 2016.

BAZZO, V. L. Profissionalidade docente na educação superior mestres ou cientistas? Anais... Seminário de Pesquisa em Educação da Região Sul, 5., 2008. Itajaí: ANPED Sul Univale, 2008. (CD-ROM).

BEZERRA, J. E. M. S. Saúde Mental de estudantes dos cursos de graduação em engenharia: experiências psicoemocionais na universidade. 2018. 127 f. Dissertação (Mestrado em Saúde Coletiva) - Centro de Ciências da Saúde da Universidade Estadual do Ceará, Universidade de Federal do Ceará. Fortaleza, 2018.

CASALE, A.; KURI, N. P.; SILVA, A. N. R. Mapas cognitivos na avaliação da Aprendizagem Baseada em Problemas. Revista Portuguesa de Educação, Minho, v. 24, n. 2, p. 243-263, 2011. 
EASTMAN, C. et al. Manual de BIM: um guia de modelagem da informação da construção para arquitetos, engenheiros, gerentes, construtores e incorporadores. Porto Alegre: Bookman, 2014.

FRAGA, É. A educação não cognitiva ganha espaço. Folha de São Paulo, São Paulo, 15 de mar. de 2015. Disponível em: $<$ https://www1.folha.uol.com.br/ilustrissima/2 015/03/1602386-a-educacao-nao-cognitivaganha-espaco.shtml>. Acesso em: 09 out. 2019.

FURTADO, A. F. Um estudo sobre o desafio do ensino de engenharia frente aos problemas econômicos, energéticos e a sustentabilidade. Revista Encontro de Pesquisa em Educação, Uberaba, v. 1, n. 1, p. 4-19, out. 2013.

GUILHERMO, O. E. P. et al. Ensino e aprendizagem de hidráulica através de um Laboratório Virtual de Aprendizagem. Revista Eletrónica de Investigación em Educación en Ciencias, Buenos Aires, v. 1, n. 2, p. 43-54, jul. 2017.

LAVADO, T. Uso da internet no Brasil cresce, e $70 \%$ da população está conectada. Portal G1, 28 de agosto de 2019. Seção de Tecnologia. Disponível em: <https://g1.globo.com/economia/tecnologia/n oticia/2019/08/28/uso-da-internet-no-brasilcresce-e-70percent-da-populacao-estaconectada.ghtml>. Acesso em: 11 out. 2019.

MASETTO, M. T. Competências pedagógicas do professor universitário. São Paulo: Summus, 2012.

MIRANDA, A. C. O.; MATOS, C. R. Potencial uso o BIM na fiscalização de obras públicas. Revista TCU, Brasília, v. 133, p. 22-31, mai./ago. 2015.

MOLISANI, A. L. Evolução do perfil didáticopedagógico do professor-engenheiro. Revista Educação e Pesquisa, São Paulo, v. 43, n. 2, p. 467-482, abr./jun. 2017.

MONTEIRO, S. et al. Alunos de excelência no ensino: comunalidades e singularidades na trajectória académica. Análise psicológica, Lisboa, v. 1, XXVII, p. 79-87, 2009.

PASSOS, F. G. et al. Diagnóstico sobre a reprovação nas disciplinas básicas dos cursos de engenharia da UNIVASF. Anais... XXXV Congresso Brasileiro de Educação em Engenharia, p. 1-16. Curitiba: UnicenP, 2007.

PAVANELO, E.; LIMA, R. Sala de Aula Invertida: a análise de uma experiência na disciplina de Cálculo I. Bolema: Boletim de Educação Matemática, Rio Claro, v. 31, n. 58, p. 739759, ago. 2017.

PAWSON, E. et al. Problem-based learning in Geography: Towards a critical assessment of its purposes, benefits and risks. Journal of Geography in Higher Education, v. 30, n. 1, p. 103-116, mar. 2006.

SACCARO, A.; FRANÇA, M. T. A.; JACINTO, P. A. Fatores Associados à Evasão no Ensino Superior Brasileiro: um estudo de análise de sobrevivência para os cursos das áreas de Ciência, Matemática e Computação e de Engenharia, Produção e Construção em instituições públicas e privadas. Estudos Econômicos, São Paulo, v. 49, n. 2, p. 337373, abr./jun. 2019.

SAEB. Sistema de Avaliação da Educação Básica, Resultados Saeb, 2017.

SENO, W. P.; BELHOT, R. V. Delimitando a fronteira para a identificação de competências para a capacitação de professores de engenharia para o ensino a distância. Revista Gestão e Produção, São Carlos, v. 16, n. 3, p. 502-514, jul.-set. 2009.

SEVERINO, A. J. Da docência no ensino superior: condições e exigências. Revista Comunicações, Piracicaba, v. 20, n. 1, p. 4352, jan./jun. 2013.

VIDAL, L. Â.; CUNHA, C. R. A reprovação nas disciplinas de física causada pela ausência de bases matemáticas nos ensinos fundamental e médio. Experiências em Ensino de Ciências, Cuiabá, v. 14, n. 1, p. 510-521, abr. 2019. 


\section{DADOS BIOGRÁFICOS DOS AUTORES}
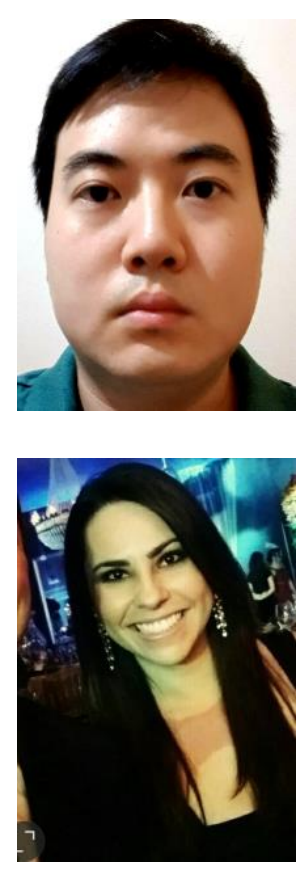

Gustavo Yoshio Matsubara - Graduado em Engenharia Civil pela Universidade Tecnológica Federal do Paraná (2015), Mestre em Engenharia Civil na área de Estruturas e Materiais pela Universidade Federal do Rio de Janeiro/COPPE (2018). Doutorando em Engenharia Civil (UFRJ/COPPE) na área de estabilidade estrutural em estruturas de aço. Atuou como professor mediador na UniCesumar e tem experiência com desenvolvimento de materiais didáticos como professor conteudista na área de estruturas de madeiras. Possui interesse nas áreas de estruturas (aço, madeira e concreto) e metodologias alternativas de ensino aplicadas à engenharia.

Tayza Cristina Nogueira Rossini - Graduada em Letras Lic. Plena (PortuguêsInglês) e Mestre na linha de pesquisa Literatura e Construção de Identidades pela Universidade Estadual de Maringá (UEM). Professora da Graduação (Presencial) e orientadora de trabalhos de conclusão de curso (TCC) de Pós-graduação do Centro Universitário de Maringá (UniCesumar), além de professora do curso de Letras da Universidade Estadual de Maringá (UEM). É, ainda, avaliadora do BASIS (Instituto Nacional de Estudos e Pesquisas Anísio Teixeira - INEP). 